\title{
Management and Outcome of Testicular Torsion
}

John Murithi ${ }^{1}$, Abidan Mwachi ${ }^{1}$, Ramadhani Abdalla ${ }^{1}$, Sundeep Chavda ${ }^{2}$

1. Coast General Hospital, Mombasa

2. Pandya Memorial Hospital, Mombasa

Correspondence to:Dr. John Murithi, P.O. Box 90062 - 80100, Mombasa, Kenya. Email: johnkinyua@gmail.com

\section{Abstract}

Background:Testicular torsion is a common cause of acute scrotum and is complicated by loss of testes. Local data on testicular torsion is limited. This study therefore aimed at determining the incidence and clinical presentation of patients with testicular torsion in our setup.Methods: This retrospective study reviewed files of 90 patients admitted to our hospital with diagnosis of testicular torsion from January 2011 to December 2015.Results: The mean age of presentation was $19 \pm 6$ years. Fifty-nine $(66 \%)$ of the patients presented with right sided torsion while $5(6 \%)$ had bilateral torsion. Acute, intermittent and missed types of torsion were seen in 59 (66\%), 19 (21\%) and 12 (13\%) respectively. The duration of symptoms from onset tosurgery was $17 \pm 4$ hours. The testis salvage rates for

\section{Introduction}

Testicular torsion results from twisting of the vessels supplying the testes with resultant ischemia (1) It is described as acute or intermittent based on the timing of presentation (2). When a patient presents with symptoms for less than 24 hours, it is referred to as acute torsion $(2,3)$. On the other hand, intermittent torsion is characterized by recurrent scrotal pain caused by intermittent torsion and detorsion of the testis (4). No definitive test is available for intermittent torsion (5). The diagnosis of missed testicular torsion is made preoperatively on ultra sound or confirmed retrospectively during scrotal exploration (5).

Management of testicular torsion involves detorsion of the testes and orchidopexy (1). Salvage rates are governed by speed of diagnosis and treatment. Delay in diagnosis or treatment can result in non-viable testes, this is described as a missed torsion (6).

\section{Methods}

This retrospective descriptive study was carried out at the Coast General Hospital in Kenya. This is a tertiary acute and intermittent torsion were $18 \%$ and $21 \%$ respectively. Conclusion: Testis salvage rates were lower in our setup compared to other studies. This could be due to delays in presentation and in diagnosis. Therefore, a high index of suspicion should always be maintained in all cases of acute scrotal pain.

Key words: Testicular, Torsion, Acute scrotum, Intermittent, Missed

Ann Afr Surg. 2017; 14(2):104-107

DOI:http://dx.doi.org/10.4314/aas.v14i2.11

(C) 2017 Author. This work is licensed under the Creative Commons Attribution 4.0 International License.

population of 3 million people (7).The hospital is staffed with emergency department medical officers surgical residents and consultant surgeons including two urologists. The initial diagnosis of testicular torsion is made by medical officers and thereafter the patients are handed over to the surgical team for definitive management. Surgery is done by the consultant surgeons assisted by the surgical resident. The patient is subsequently followed up at the surgical clinics manned by both the residents and surgeons (8).

We reviewed the files of all the patients presenting to the hospital with testicular torsion from January 2011 to December 2015. We excluded patients with incomplete data. The variables collected included: patient's age, duration of symptoms from onset to surgery, intraoperative findings as to whether the testes were salvageable or not and whether surgery was done as an elective or emergency procedure. Data collected were analyzed using Microsoft Excel spread sheet.Ethical approval was obtained from the hospital ethics and review board. 


\section{Results}

Ninety patients over a five-year period were identified from the hospital registry and their files retrieved. The mean age of presentation was $19 \pm 6$ years, with a peak incidence between $11-21$ years (Figure 1).

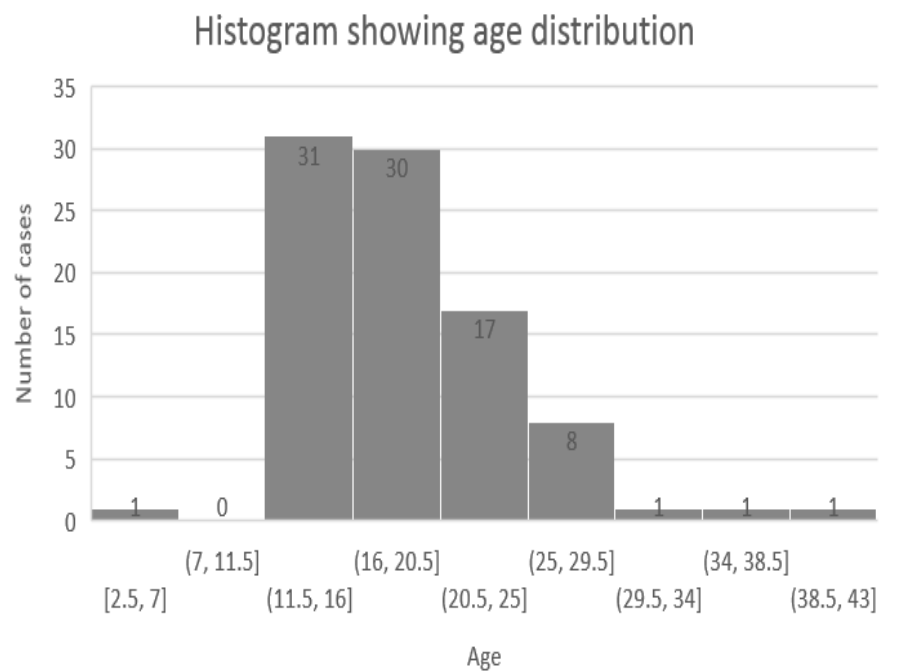

Figure 1: Distribution of testicular torsion with age

Fifty-nine $(66 \%)$ of the patients presented with right sided torsion while $5(6 \%)$ had bilateral torsion. The rest had left sided torsion. Acute, intermittent and missed types of torsion were seen in 59 (66\%), 19 (21\%) and $12(13 \%)$ respectively. The duration of symptoms from onset to surgery was $17 \pm 4$ hours for acute torsion while that for intermittent and missed torsion $19 \pm 14$ days. All 59 cases of acute torsion and $2(16 \%)$ cases of missed torsion underwent emergency surgeries. The rest (10 cases of missed torsion and all cases of intermittent torsion) underwent elective procedures. The testis salvage rates for acute and intermittent torsion were $18 \%$ and $21 \%$ respectively. None of the patients with missed torsion had salvageable testes. Sixty-five (72\%) patients required orchiectomy. A salvage rate of $18 \%$ was achieved in the patients with acute torsion.

\section{Discussion}

Age at presentation was $19 \pm 6$ with a peak incidence of 11 to 21 years. This is comparable to previous studies $(2,9)$. A study conducted in a similar setup found a mean age of 17years (1) which is similar to our findings. Testicular torsion is found mostly in men of less than 25 years with an incidence of 1 in 4000 (10). This presentation in young adult males is thought to be due to impulsive contraction of the cremaster muscles and developmental changes occurring in puberty such as increased weight of the testes (3). Presence of an attachment of the tunica vaginalis higher than normal in what is known as a bell clapper deformity is believed to further increase susceptibility in this age group $(10,11)$. Multiple cases have been documented in literature of testicular torsion in the same family through generations but no mutations have been proven to be responsible (12). Most patients in our setup present with right sided torsion $66 \%$, with bilateral torsion seen in $6 \%$ of the patients. This is departure from available literature which reports a left sided predominance $(12,13$,$) . No$ literature could be found on anatomic or embryological reason for this. Further research is required to establish the true predominance. The number of patients in this study was also small $(n=90)$ which may contribute to the variance.Acute testicular torsion was the most common presentation in patients presenting at our facility at $59(66 \%)$ patients. A similar study conducted in the same setup found 42 patients with acute torsion over the course of 12 years (1). The increased number of patients identified with acute torsion stems from increased awareness created by the preceding study as the findings were made public following publication of the study. Statistics comparing the three types of torsion is unavailable, incidence of acute testicular torsion in patients presenting with acute scrotum is reported at 34 $\%$ in one series (14) where a torted cyst of Morgagni was found to be a more common cause of acute scrotum. Missed torsion accounted for $13 \%(\mathrm{~N}=15)$ of the cases. Causes of missed torsion was postulated to be due inadequate clinical examination and over reliance on imaging as all patients presenting with acute scrotum were subjected to an ultrasound, which delayed surgical intervention. Presence of a high riding testicle with absent cremasteric reflex warrants immediate surgical exploration without imaging studies (15, 16).Intermittent torsion was seen in $21 \%$ of the patients. Intermittent torsion is caused by twisting of the spermatic cord due to the same factors as those of acute torsion but in this case untwisting occurs before infarction can occur (17). There was a low (18\%) salvage rate among the patients who presented with testicular torsion. Previous studies have reported a salvage rate of between $14 \%$ and $55 \%(1,19,20)$. This could be attributed to delayed surgical intervention (17 \pm 4 hours) and delay in recognizing testicular torsion as 
evidenced by the rate of missed torsion reported in this study. Accurate diagnosis and timely intervention such as surgery done within 4 hours improves the salvage rates (18). Patients presenting after 24 hours have elapsed have a less than $10 \%$ chance of salvage $(10,12)$ with optimal results expected if surgery is done within four to eight hours (16). Although salvage rates for acute torsion in this study were low, a similar study conducted in this setup (1) showed salvages rates of $14 \%$. This improvement may be attributed to sensitization that followed the previous study. Of note is that the decision as to whether the testes were salvageable or not were made on table which could have resulted in viable testes to be removed. Normal testosterone values and normal inter-testicular blood flow has been documented in patients in whom orchidectomy was not performed in spite of testes appearing non viable during surgery (12). Absence of information on time from diagnosis to intervention time was a major drawback in our study. Time from onset of symptoms to surgery plays a major role in outcome (11, $13,15)$ and subsequent complications. Subsequent studies will therefore have to focus on this aspect of testicular torsion as it has a direct impact on outcome.

Previous studies in this setup recommended enhanced public awareness regarding the potential consequences of acute scrotal pain in addition to clinician, parental, teacher, teenage and adult male education in an attempt to mitigate sequelae of testicular torsion (1). This may have played a role in the higher number of patients $(n=90)$ observed over a similar 5-year period and slight improvement in salvage rates (18\% versus $14 \%)$.

\section{Conclusion}

This study reports a low testis salvage rate among patients presenting with either acute or intermittent testicular torsion. This could be attributable to delay in presentation or in diagnosis. The findings of this study can therefore be used to sensitization primary care givers and persons at risk of the pathology.

\section{References}

1. Maranya GA, Mwero BJ, Kinyanjui GM, et al. Dismal Salvage Rates of Testicular Torsion: A Call to Action. Ann Afr Med, 2011;8: 8-10

2. Beni-Israel T, Goldman M, Bar Chaim S, et al. Clinical Predictors for Testicular Torsion as Seen in the Pediatric ED. Am J Emerg Med. 2010; 28(7):786-9

3. Nickel JC, Tiechman JM, Gregoine $M$, et al. Prevalence, Diagnosis, Characterisation and Treatment of Prostatitis, Interstitial Cystitis and Epidydimitis in Outpatient Urological Practice: The Canadian PIE Study. Urology 2005; 66(5)935-940

4. Pearce T, Islam S, O' Flynn KJ. Suspected Testicular Torsion: A Survey of Clinical Practice in North West England. J R Soc Med. 2002; 95: 247.

5. Nevo A, Mano R, Sivan B, et al. Missed Torsion of the Spermatic Cord: A Common Yet Underreported Event. Urology.2017; 102: 202-206

6. Quddus MB, Mahmud SM. Testicular Torsion: A Diagnosis not to be Missed. J Pak Med Assoc. 2011; 61(4):391-2

7. MSH, AFS, CGH: Research International East Africa Limited. Coast Province General Hospital report 2015

8. Lee SM, Huh JS, BaekM, et al. A Nationwide Epidemiological Study of Testicular Torsion in Korea. J Korean Med Sci. 2014; 29(12): 1684-1687.

9. Cattolica EV, Karol JB, Rankin KN, et al. High Testicular Salvage Rate in Torsion of the Spermatic Cord. J Urol. 1982; 128(1):66-8.

10. Eaton SH, Cendron MA, Estrada CR, et al. Intermittent Testicular Torsion: Diagnostic Features and Management. J Urol. 2005; 174 (4 prt 2):15321535

11. Fana R, Zhangb J, Chenga L, et al. Abnormalities of the Testes and Scrotum and their Surgical Management. Welsh Urology 9th Edition Philadelphia, PA: WB Saunders; 2012. 642-5

12. Feher AM, Zoltan B. A Review of Main Controversial Aspects of Acute Testicular Torsion. Journal of Acute Disease 2016; 5(1):1-8

13. Ringdahl E, Teague L. Testicular Torsion. Am Fam Physician. 2006 Nov 15. 74(10):1739-43.

14. Kar A, Ozden E, Yakupoglu YK, et al. Experimental Unilateral Spermatic Cord Torsion: The Effect of Polypolymerase Enzyme Inhibitor on Histopathological and Biochemical Changes in Early and Late Periods in the Ipsilateral and Contralateral Testicles. Urology. 2010; 76 (2): 507. E1-5 
15. Tajchner L, Larkin JO, Bourke MG, et al. Management of the Acute Scrotum in a District General Hospital: A 10-Year Experience. Scient. Worl. J. 2009; 9: 281-286.

16. Cost NG, Bush NC, Barber TD, et al. Pediatric Testicular Torsion: Demographics of National Orchiopexy Versus Orchiectomy rates. J Urol. 2011;185(6 Suppl):2459-63

17. Sharp VJ, Kieran K. Testicular Torsion: Diagnosis, Evaluation and Management. Am Fam Physician. 2013; 88(12):835-840.

18. Barker K, Raper FP. Torsion of the Testis. Br J Urol 1964; 36:35-41.

19. Ramachandra P, Palazzi KL, Holmes NM, et al. Factors Influencing Rate of Testicular Salvage in Acute Testicular Torsion at a Tertiary Pediatric Center. West J Emerg Med. 2015; 16(1): 190- 194.

20. Magoha GA. Testicular Torsion Salvage Rate in Nigerians in Lagos. East Afr Med J. 1989; 66(5):324-7. 